Network Working Group

Request for Comments: 5303

Obsoletes: 3373

Category: Standards Track
D. Katz

Juniper Networks

R. Saluja

Tenet Technologies

D. Eastlake 3rd

Eastlake Enterprises

October 2008

\title{
Three-Way Handshake for IS-IS Point-to-Point Adjacencies
}

Status of This Memo

This document specifies an Internet standards track protocol for the Internet community, and requests discussion and suggestions for improvements. Please refer to the current edition of the "Internet Official Protocol Standards" (STD 1) for the standardization state and status of this protocol. Distribution of this memo is unlimited.

\section{Abstract}

The IS-IS routing protocol (Intermediate system to Intermediate System, ISO 10589) requires reliable protocols at the link layer for point-to-point links. As a result, it does not use a three-way handshake when establishing adjacencies on point-to-point media. This paper defines a backward-compatible extension to the protocol that provides for a three-way handshake. It is fully interoperable with systems that do not support the extension.

Additionally, the extension allows the robust operation of more than 256 point-to-point links on a single router.

This extension has been implemented by multiple router vendors; this paper is provided to the Internet community in order to allow interoperable implementations to be built by other vendors. 
Table of Contents

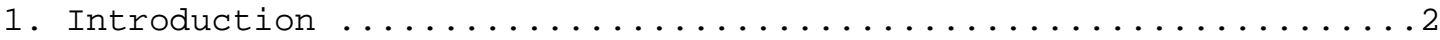

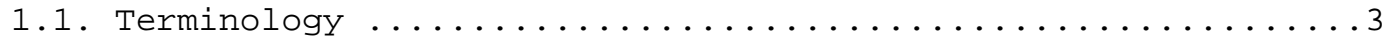

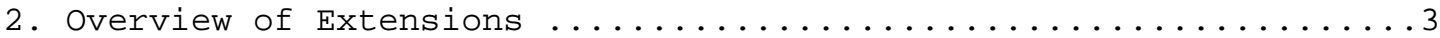

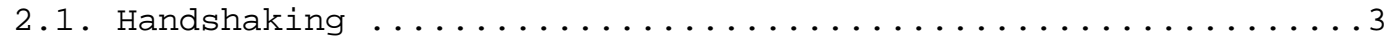

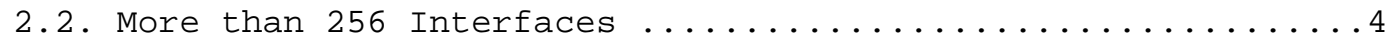

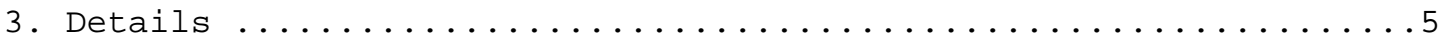

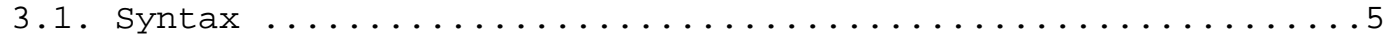

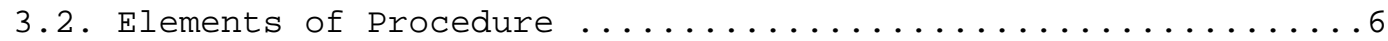

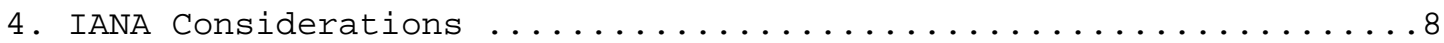

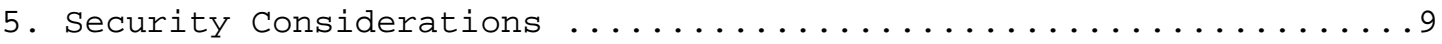

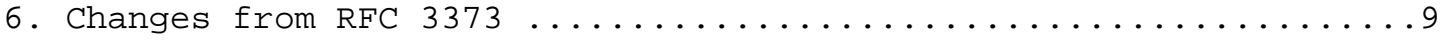

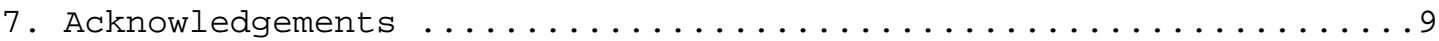

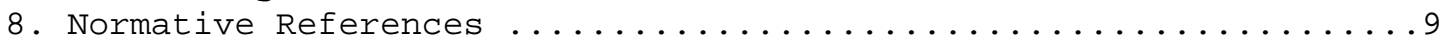

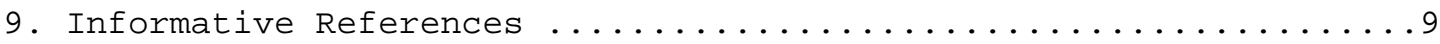

\section{Introduction}

The IS-IS protocol [ISIS] assumes certain requirements stated in ISO 10589 (section 6.7.2) for the operation of IS-IS over point-to-point links and hence provides only a two-way handshake when establishing adjacencies on point-to-point links. The protocol does not operate correctly if these subnetwork requirements for point-to-point links are not met. The basic mechanism defined in the standard is that each side declares the other side to be reachable if a Hello packet is heard from it. Once this occurs, each side then sends a complete Sequence Number PDU (CSNP) to trigger database synchronization.

Three failure modes are known. First, if the link goes down and then comes back up, or one of the systems restarts, and the CSNP packet is lost, and the network has a cut set of one through the link, the link state databases on either side of the link will not synchronize for a full Link state Protocol Data Unit (LSP) refresh period (up to 18 hours).

A second, more serious failure is that if the link fails in only one direction, the failure will only be detected by one of the systems. Normally only one of the two systems will announce the adjacency in its link state packets, and the SPF algorithm will thus ignore the link. However, if there are two parallel links between systems and one of them fails in one direction, SPF will still calculate paths between the two systems, and the system that does not notice the failure will attempt to pass traffic down the failed link (in the direction that does not work).

The third issue is that on some physical layers, the interconnectivity between endpoints can change without causing a 
link-layer-down condition. In this case, a system may receive packets that are actually destined for a different system (or a different link on the same system). The receiving system may end up thinking that it has an adjacency with the remote system when in fact the remote system is adjacent with a third system.

The solution proposed here ensures correct operation of the protocol over unreliable point-to-point links. As part of the solution to the three-way handshaking issue, a method is defined to remove the limitation of 255 point-to-point interfaces imposed by IS-IS [ISIS]. This method is more robust than the ad hoc methods currently in use.

\subsection{Terminology}

The key words "MUST", "MUST NOT", "REQUIRED", "SHALL", "SHALL NOT", "SHOULD", "SHOULD NOT", "RECOMMENDED", "MAY", and "OPTIONAL" in this document are to be interpreted as described in [RFC2119].

2. Overview of Extensions

This section provides a general overview of the three-way handshaking provided and how more than 256 interfaces are handled.

\subsection{Handshaking}

The intent is to provide a three-way handshake for point-to-point adjacency establishment in a backward-compatible fashion. This is done by providing an optional mechanism that allows each system to report its adjacency three-way state, thus allowing a system to only declare an adjacency to be up if it knows that the other system is receiving its IS-IS Hello (IIH) packets.

The adjacency three-way state can be one of the following types:

Down

This is the initial point-to-point adjacency three-way state. The system has not received any IIH packet containing the three-way handshake option on this point-to-point circuit.

Initializing

The system has received an IIH packet containing the three-way handshake option from a neighbor but does not know whether the neighbor is receiving its IIH packet.

Up The system knows that the neighbor is receiving its IIH packets. 
The adjacency three-way state that is reported by this mechanism is not equal or equivalent to the adjacency state that is described in ISO 10589 [ISIS]. If this mechanism is supported, then an adjacency may have two states, its state as defined in ISO 10589 [ISIS], and its three-way state. For example, according to ISO 10589, receipt of an Intermediate system Hello (ISH) will cause an adjacency to go to Initializing state; however, receipt of an ISH will have no effect on the three-way state of an adjacency, which remains firmly Down until it receives an IIH from a neighbor that contains the three-way handshaking option.

In addition, the neighbor's system ID and (newly defined) extended circuit ID are reported in order to detect the case where the same stream is being received by multiple systems (only one of which can talk back).

The mechanism is quite similar to the one defined in the Netware Link Services Protocol (NLSP) [NetLink], a variant of IS-IS used for routing Internetwork Packet Exchange (IPX) traffic. The difference between this mechanism and the one used in NLSP is the location where the information is carried (NLSP uses two of the reserved bits in the IIH header, whereas this solution adds a separate option to the IIH), and the presence of the neighbor's system ID and circuit ID. In theory, using the reserved header bits should be backward compatible, since systems are supposed to ignore them. However, it was felt that this was risky, as the use of untested mechanisms such as this have led to problems in the past in other protocols. New option codes, on the other hand, have been demonstrated to work properly, as the deployment of Integrated IS-IS for IP [RFC1195] has done exactly this.

The new mechanism only comes into play when the remote system includes the new option in its IIH packet; if the option is not present, it is assumed that the system does not support the new mechanism, and so the old procedures are used.

\subsection{More than 256 Interfaces}

The IS-IS specification has an implicit limit of 256 interfaces, as constrained by the eight-bit Circuit ID field carried in various packets. Moderately clever implementers have realized that the only true constraint is that of 256 LAN interfaces, and for that matter only 256 LAN interfaces for which a system is the Designated IS. This is because the only place that the circuit ID is advertised in LSPS is in the pseudo-node LSP ID. 
Implementers have treated the point-to-point circuit ID number space as being independent from that of the LAN interfaces, since these circuit IDs appear only in IIH PDUs and are only used for detection of a change in identity at the other end of a link. More than 256 point-to-point interfaces have been supported by sending the same circuit ID on multiple interfaces. This reduces the robustness of the ID change detection algorithm, since it would then be possible to switch links between interfaces on a system without detecting the change.

Since the circuit ID is an integral part of the new handshaking mechanism, a backward-compatible mechanism for expanding the circuit ID number space is included in this specification.

3. Details

The detailed syntax and procedures for this IS-IS option are given below.

\subsection{Syntax}

A new IS-IS Option type, "Point-to-Point Three-Way Adjacency", is defined:

Type - 0xF0 (decimal 240)

Length - total length of the value field (1 to 17 octets)

Value -

No. of Octets

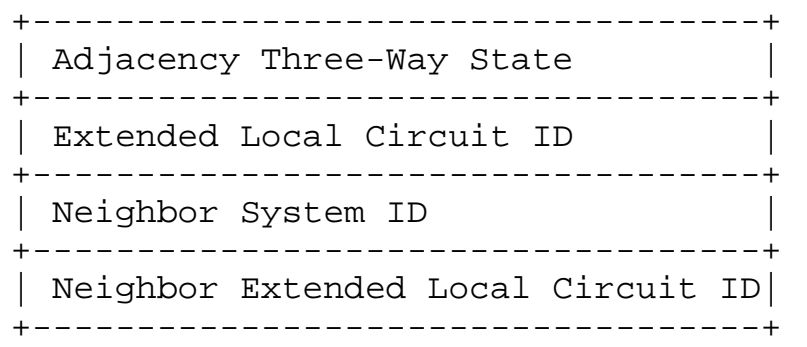

Adjacency Three-Way state

The adjacency three-way state of the point-to-point adjacency. The following values are defined:

$$
\begin{aligned}
& 0-\text { Up } \\
& 1-\text { Initializing } \\
& 2-\quad \text { Down }
\end{aligned}
$$


Extended Local Circuit ID

Unique ID assigned to this circuit when it is created by this Intermediate system.

Neighbor system ID System ID of neighboring Intermediate system if known. The length of this field is equal to "ID Length" of the IIH PDU described in "Point-to-point IS to IS hello PDU" (section 9.7 of [ISIS]).

Neighbor Extended Local Circuit ID

Extended Local Circuit ID of the other end of the point-to-point adjacency if known.

Any system that supports this mechanism SHALL include this option in its Point-to-Point IIH packets.

Any system that does not understand this option SHALL ignore it, and (of course) SHALL NOT include it in its own IIH packets.

Any system that supports this mechanism MUST include the Adjacency Three-Way state field in this option. The other fields in this option SHOULD be included as explained below in section 3.2 .

Any system that is able to process this option SHALL follow the procedures below.

\subsection{Elements of Procedure}

The new handshake procedure is added to the IS-IS point-to-point IIH state machine after the PDU acceptance tests have been performed.

Although the extended circuit ID is only used in the context of the three-way handshake, it is worth noting that it effectively protects against the unlikely event where a link is moved to another interface on a system that has the same local circuit ID, because the received PDUs will be ignored (via the checks defined below) and the existing adjacency will fail.

Add a clause e) to the end of "Receiving ISH PDUs by an intermediate system" (section 8.2.2 of [ISIS]):

Set the state to be reported in the Adjacency Three-Way state field of the Point-to-Point Three-Way Adjacency option to Down. 
Add a clause e) to the end of "Sending point-to-point IIH PDUs" (section 8.2 .3 of [ISIS]):

The IS SHALL include the Point-to-Point Three-Way Adjacency option in the transmitted Point-to-Point IIH PDU. The current three-way state of the adjacency with its neighbor on the link (as defined in new section 8.2.4.1.1 introduced later in the document) SHALL be reported in the Adjacency Three-Way state field. If no adjacency exists, the state SHALL be reported as Down.

The Extended Local Circuit ID field SHALL contain a value assigned by this IS when the circuit is created. This value SHALL be unique among all the circuits of this Intermediate system. The value is not necessarily related to that carried in the Local Circuit ID field of the IIH PDU.

If the system ID and Extended Local Circuit ID of the neighboring system are known (in adjacency three-way state Initializing or Up), the neighbor's system ID SHALL be reported in the Neighbor System ID field, and the neighbor's Extended Local Circuit ID SHALL be reported in the Neighbor Extended Local Circuit ID field.

Add a section 8.2.4.1.1, "Three-Way Handshake", immediately prior to "IIH PDU Processing" (section 8.2.4.2 of [ISIS]):

A received Point-to-Point IIH PDU may or may not contain the Point-to-Point Three-Way Adjacency option. If it does not, the link is assumed to be functional in both directions, and the procedures described in section 8.2.4.2 are followed.

If the option is present and contains invalid Adjacency Three-Way State, the PDU SHALL be discarded and no further action is taken.

If the option with a valid Adjacency Three-Way state is present, the Neighbor System ID and Neighbor Extended Local Circuit ID fields, if present, SHALL be examined. If they are present, and the Neighbor system ID contained therein does not match the local system's ID, or the Neighbor Extended Local Circuit ID does not match the local system's extended circuit ID, the PDU SHALL be discarded and no further action is taken.

If the Neighbor System ID and Neighbor Extended Local Circuit ID fields match those of the local system, or are not present, the procedures described in section 8.2.4.2 are followed with the following changes: 
a) In section 8.2.4.2 a and b, the action "Up" from state tables $5,6,7$, and 8 may create a new adjacency but the three-way state of the adjacency SHALL be Down.

b) If the action taken from section 8.2.4.2 a or b is "Up" or "Accept", the IS SHALL perform the action indicated by the new adjacency three-way state table below, based on the current adjacency three-way state and the received Adjacency Three-Way State value from the option. (Note that the procedure works properly if neither field is ever included. This provides backward compatibility to an earlier version of this option.)

\begin{tabular}{|c|c|c|c|c|}
\hline & & $\begin{array}{c}\text { Received Ac } \\
\text { Down }\end{array}$ & $\begin{array}{l}\text { Three-Way } \\
\text { ializing }\end{array}$ & $\begin{array}{l}\text { State } \\
\text { Up }\end{array}$ \\
\hline & Down & Initialize & Up & Down \\
\hline $\begin{array}{l}\text { Adj. } \\
\text { Three- }\end{array}$ & Initializing & Initialize & Up & Up \\
\hline $\begin{array}{l}\text { Way } \\
\text { State }\end{array}$ & Up & Initialize & Accept & Accept \\
\hline
\end{tabular}

Adjacency Three-Way state Table

If the new action is "Down", an adjacencystatechange (Down) event is generated with the reason "Neighbor restarted" and the adjacency SHALL be deleted.

If the new action is "Initialize", no event is generated and the adjacency three-way state SHALL be set to "Initializing".

If the new action is "Up", an adjacencystatechange(Up) event is generated.

c) Skip section 8.2.4.2 c and d.

d) If the new action is "Initialize", "Up", or "Accept", follow section $8.2 .4 .2 \mathrm{e}$.

4. IANA Considerations

This document specifies IS-IS Option 240 (0xF0), which has already been allocated. See [RFC3359]. 


\section{Security Considerations}

This document raises no new security issues for IS-IS. IS-IS security may be used to secure the IS-IS messages discussed here. See [RFC5304].

6. Changes from RFC 3373

This document is a minor edit of [RFC3373] with the intent of advancing it from Informational to Standards Track. It also updates the ISP 10589 reference to refer to the current "2002" version.

7. Acknowledgements

Thanks to Tony Li, Henk Smit, Naiming Shen, Dave Ward, Jeff Learman, Les Ginsberg, and Philip Christian for their contributions to the document.

8. Normative References

[ISIS] ISO, "Intermediate system to Intermediate system intradomain routeing information exchange protocol for use in conjunction with the protocol for providing the connectionless-mode network service (ISO 8473)", International Standard 10589:2002, Second Edition, 2002 .

[NetLink] "Netware Link Services Protocol Specification, Version 1.0", Novell, Inc., February 1994.

[RFC1195] Callon, R., "Use of OSI IS-IS for routing in TCP/IP and dual environments", RFC 1195, December 1990.

[RFC2119] Bradner, S., "Key words for use in RFCs to Indicate Requirement Levels", BCP 14, RFC 2119, March 1997.

9. Informative References

[RFC3373] Katz, D. and R. Saluja, "Three-Way Handshake for Intermediate system to Intermediate system (IS-IS) Pointto-Point Adjacencies", RFC 3373, september 2002.

[RFC3359] Przygienda, T., "Reserved Type, Length and Value (TLV) Codepoints in Intermediate system to Intermediate system", RFC 3359, August 2002 .

[RFC5304] Li, T. and R. Atkinson, "IS-IS Cryptographic Authentication", RFC 5304, October 2008. 


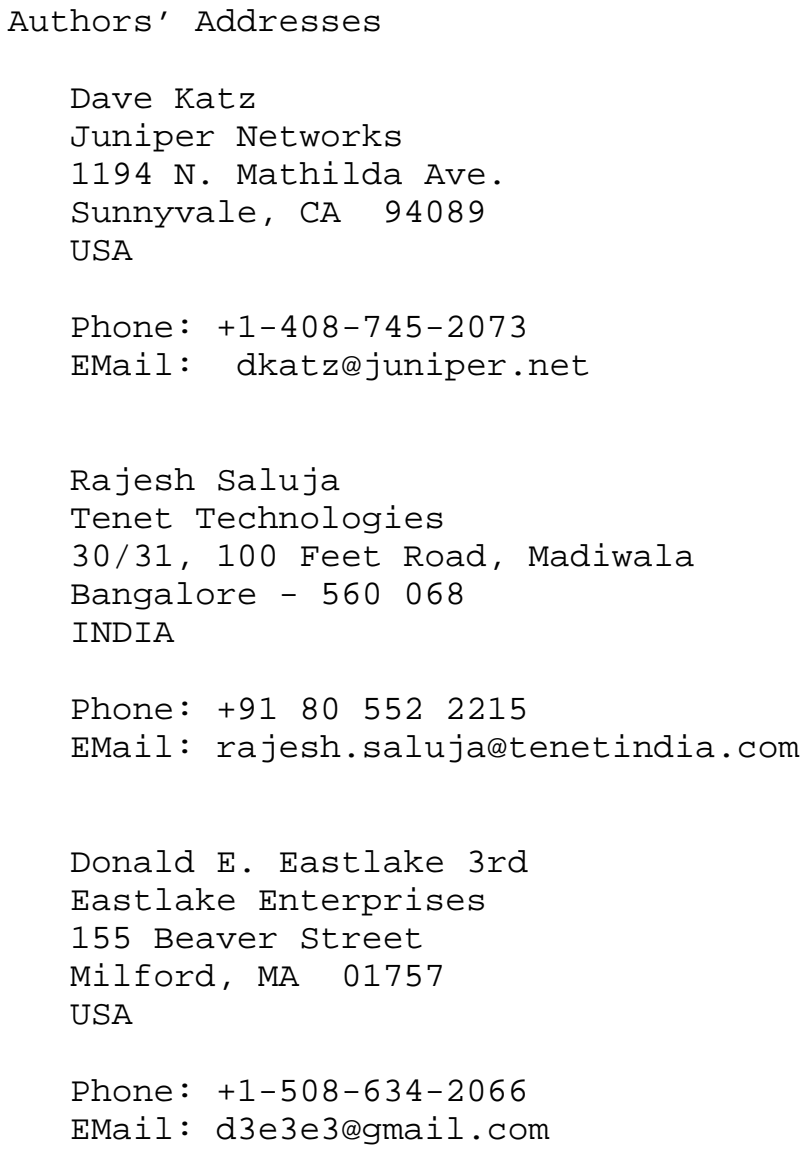


Full Copyright statement

Copyright (C) The IETF Trust (2008).

This document is subject to the rights, licenses and restrictions contained in BCP 78, and except as set forth therein, the authors retain all their rights.

This document and the information contained herein are provided on an "AS IS" basis and THE CONTRIBUTOR, THE ORGANIZATION HE/SHE REPRESENTS OR IS SPONSORED BY (IF ANY), THE INTERNET SOCIETY, THE IETF TRUST AND THE INTERNET ENGINEERING TASK FORCE DISCLAIM ALL WARRANTIES, EXPRESS OR IMPLIED, INCLUDING BUT NOT LIMITED TO ANY WARRANTY THAT THE USE OF THE INFORMATION HEREIN WILL NOT INFRINGE ANY RIGHTS OR ANY IMPLIED WARRANTIES OF MERCHANTABILITY OR FITNESS FOR A PARTICULAR PURPOSE.

Intellectual Property

The IETF takes no position regarding the validity or scope of any Intellectual Property Rights or other rights that might be claimed to pertain to the implementation or use of the technology described in this document or the extent to which any license under such rights might or might not be available; nor does it represent that it has made any independent effort to identify any such rights. Information on the procedures with respect to rights in RFC documents can be found in BCP 78 and BCP 79 .

Copies of IPR disclosures made to the IETF Secretariat and any assurances of licenses to be made available, or the result of an attempt made to obtain a general license or permission for the use of such proprietary rights by implementers or users of this specification can be obtained from the IETF on-line IPR repository at http://www.ietf.org/ipr.

The IETF invites any interested party to bring to its attention any copyrights, patents or patent applications, or other proprietary rights that may cover technology that may be required to implement this standard. Please address the information to the IETF at ietf-ipreietf.org. 